\title{
Comunidade de Conhecimentos e Práticas em Promoção à Saúde de Minas Gerais: compartilhar para promover saúde
}

\section{Community of Knowledge and Practices in Health Promotion of Minas Gerais: sharing to promote health}

Fernanda Gonçalves de Souza ${ }^{1 *}$, Vanessa Vidal de Castro ${ }^{2}$, Carolina Guimarães Marra Nascimento $^{2}$, Daniela Souzalima Campos ${ }^{2}$, Nathalia Ribeiro Mota Beltrão ${ }^{2}$, Barbara Quintela Zanette Reis ${ }^{2}$, Cristiane Roberta Pinto Tomaz ${ }^{2}$, Wanessa Debôrtoli de Miranda ${ }^{1}$

\begin{abstract}
RESUMO
Trata-se de um relato de experiência, a partir das vivências durante o Estágio Curricular Não-Obrigatório na Diretoria de Promoção à Saúde na Secretaria de Estado de Saúde de Minas Gerais (SES-MG) incluso na Superintendência da Atenção Primária à Saúde. Tendo como objetivo apresentar as experiências para a continuidade do acervo virtual "Comunidade de Conhecimentos e Práticas em Promoção à Saúde de Minas Gerais", e o formulário de "Educação Permanente em Saúde durante a pandemia pela Covid-19”. Nota-se, a importância da Educação Permanente em Saúde, temática da Política Estadual de Promoção à Saúde do Estado de Minas Gerais, na construção de espaços para aprendizagem e troca de conheicmento em saúde considerando as necessidades elencadas de cada território. Além disso, a importâcia da participação discente na construção de intrusitivos e ações estratégicas para continuidade das atividades de promoção à saúde, e possibilitando o desenvolvimento de competências e habilidade em gestão dos serviços de saúde.
\end{abstract}

Palavras-chave: Promoção da Saúde; Educação Permanente; Comunicação em Saúde;

\begin{abstract}
This is an experience report, based on experiences during the Non-Mandatory Curricular Internship at the Health Promotion Board of the Minas Gerais State Health Department (SES-MG) included in the Superintendence of Primary Health Care. With the objective of presenting the experiences for the continuity of the virtual collection "Community of Knowledge and Practices in Health Promotion of Minas Gerais", and the form of "Permanent Education in Health during the pandemic by Covid-19". Note the importance of Permanent Education in Health, theme of the State Policy for Health Promotion of the State of Minas Gerais, in the construction of spaces for learning and exchange of knowledge in health considering the listed needs of each territory. In addition, the importance of student participation in the construction of intrusive and strategic actions for the continuity of health promotion activities, and enabling the development of skills and ability in the management of health services.
\end{abstract}

Keywords: Health Policy; Permanent Education; Health Communication;

\footnotetext{
${ }^{1}$ Universidade Federal de Minas Gerais.

*E-mail: souza.fergn@gmail.com

${ }^{2}$ Secretaria de Estado de Saúde de Minas Gerais, Cidade Administrativa
} 


\section{INTRODUÇÃO}

A Secretaria de Estado de Saúde de Minas Gerais (SES-MG) é responsável pela coordenação e planejamento do Sistema Único de Saúde (SUS) em todo o Estado, regendo-se pelo Decreto 47.769 de 28 de novembro de 2019. Atuando no repasse de recursos e estratégias para o fortalecimento da saúde pública nos 853 municípios mineiros, no âmbito da assistência, prevenção e promoção da saúde (MINAS GERAIS, 2021a).

Além disso, a SES-MG tem como missão desenvolver, fomentar e regular as políticas de saúde, estabelecendo as redes de atenção à saúde em todas as regiões, e promovendo o acesso a toda população mineira aos serviços de saúde. E pensando na integração ensino-serviço, e as possíveis contribuições no processo de formação profissional na área da saúde, a SES-MG desenvolveu o Programa de Estágio, em consonância com as diretrizes e princípios do SUS (MINAS GERAIS, 2020; MINAS GERAIS, 2021b).

O Estágio Curricular Não-Obrigatório (ECNO) na Diretoria de Promoção à Saúde (DPS) inclusa na Superintendência da Atenção Primária à Saúde, possibilita o desenvolvimento de estratégias sistematizadas que contribuam para adoção de práticas saudáveis, ou seja, a promoção da cultura de paz, valorização de saberes populares e tradicionais, atuação na formação e educação permanente em saúde, alimentação adequada, saudável e sustentável, de práticas corporais e atividades físicas; e no controle do álcool, tabaco e outras drogas (MINAS GERAIS, 2017).

O desenvolvimento dessas estratégias tem sido desafiador no contexto da pandemia da Covid-19, doença causada pelo novo coronavírus (Sars-CoV-2) que apresenta um espectro clínico variando de infecções assintomáticas a quadros graves (XU Z et al., 2020). A Organização Mundial de Saúde (OMS) destaca que $80 \%$ dos pacientes com a doença podem ser assintomáticos ou oligossintomáticos, e aproximadamente $20 \%$ dos casos detectados requer atendimento hospitalar, mas ainda, cerca de $5 \%$ podem necessitar de suporte ventilatório (MINAS GERAIS, 2021c apud OMS [202-]).

A pandemia tem se caracterizado como um dos maiores desafios sanitários do século, devido à falta de informações técnico-científicas, a transmissibilidade e o espectro clínico, ou seja, demandando a reorganização dos serviços de saúde para o enfrentamento. Nesse sentido, a continuidade das ações de promoção à saúde torna-se uma estratégia 
fundamental para manutenção da saúde e evitando a agudização das condições de saúde das pessoas com doenças crônicas (XU Z et al., 2020; MINAS GERAIS, 2021d).

A partir da necessidade de medidas de contenção da pandemia, a DPS em parceria com a Comunicação Social da SES tem trabalhado para apoiar as regionais de saúde e os municípios na oferta das ações de promoção da saúde, respeitando as orientações de isolamento social. Tal apoio se deu por meio da construção do Guia Orientador da Atenção Primária de Minas Gerais para o enfrentamento da pandemia de COVID-19, Guias de Saúde Mental diante a pandemia, a série "Fique em Casa com PICS" e o e-book: Ações de promoção da saúde em tempos de pandemia pelo COVID-19 (MINAS GERAIS, 2021e).

\section{OBJETIVO GERAL}

Apresentar as experiências durante o desenvolvimento de ações para a continuidade do acervo virtual "Comunidade de Conhecimentos e Práticas em Promoção à Saúde de Minas Gerais", e o formulário de "Educação Permanente em Saúde durante a pandemia pela Covid-19”.

\section{METODOLOGIA}

Trata-se de um relato de experiência de caráter descritivo, produzido a partir da vivência da discente durante o Estágio Curricular Não-Obrigatório na Diretoria de Promoção à Saúde. O estágio tem como objetivo oportunizar uma experiência profissional no setor público, assim a exposição aos desafios inerentes à realidade laboral contribui para o desenvolvimento de competências, aptidões e atitudes importantes para a vida profissional e pessoal (MINAS GERAIS, 2021b).

Os dados foram coletados a partir da observação participante da discente vivenciando um contexto de realidade, que para Correia (1999) e Spradley (1980), é caracterizada pela compreensão dos acontecimentos e suas consequências mediante o contato permanente e direto do autor com o investigado em seu cenário cultural de inserção. Além de possibilitar a atribuição de significado e descobrir a realidade para as vivências investigadas.

A participação da discente no ECNO deu-se sob supervisão da Diretora de Promoção à Saúde e com os servidores da Coordenadoria da Alimentação Saudável e Adequada e Atividade Física (CAAF) em todas as fases do desenvolvimento 
do estágio, englobando desde o desenvolvimento de atividades de compilação de dados até o acompanhamento dos programas Bolsa Família, Sistema de Vigilância Alimentar e Nutricional (SISVAN), programas de prevenção de carência de micronutrientes (PNSVA e PNSF), controle do tabagismo, Estratégia Amamenta e Alimenta Brasil (EAAB) e programa Academia da Saúde no estado de Minas Gerais (MG).

Nesse processo, a discente atuou no planejamento e desenvolvimento de ações para continuidade do acervo virtual de relatos de experiências e conhecimentos sobre promoção à saúde, intitulado Comunidade de Conhecimentos e Práticas em Promoção à Saúde de Minas Gerais, e o formulário de Educação Permanente em Saúde.

Para registrar as observações e impressões das atividades desenvolvidas, a discente utilizou documento desenvolvido no Google Docs no formato de caderno de registro adaptado, seguindo os preceitos do diário de campo, no período de março de 2020 até maio de 2021.

\section{DISCUSSÃO E RESULTADOS}

A Diretoria de Promoção à Saúde desenvolveu o hotsite Vida Saudável com objetivo de estimular a adoção de práticas sociais e de saúde pela população, contribuindo para expansão da Política Estadual de Promoção à Saúde do Estado de Minas Gerais (POEPS), e promovendo o sentimento de bem-estar na busca por modos de viver mais saudáveis e por mais qualidade de vida (MINAS GERAIS, 2021f).

A POEPS, publicada em 2016, tem como objetivo promover e incentivar o desenvolvimento de ações intra e intersetoriais que favoreçam a equidade, a melhoria das condições e modos de viver, que estimulem o empoderamento dos indivíduos e comunidades de modo a ampliar a potencialidade da saúde individual, coletiva e a participação social, buscando reduzir as desigualdades, vulnerabilidades e riscos à saúde através da atuação sobre os determinantes sociais, econômicos, políticos, culturais e ambientais como forma de fomentar indivíduos/comunidades ativos sobre a produção da sua saúde (MINAS GERAIS, 2017).

Além disso, a POEPS aborda a Educação Permanente em Saúde (EPS) como temática de referência para a formação de agendas de promoção da saúde e adoção de estratégias, operando em consonância com os princípios e valores do Sistema Único de Saúde (SUS). A POEPS destaca, ainda, como competência das Superintendências/Gerências Regionais de Saúde da Secretaria de Estado de Saúde: 
"Incentivar, promover e realizar ações de educação permanente dos gestores e trabalhadores do sistema municipal de saúde para o desenvolvimento das ações de promoção da saúde" (MINAS GERAIS, 2017).

Outrossim, visando a divulgação no Vida Saudável das experiências, apresentações e materiais técnicos selecionados desenvolvidos pelos municípios, pelas Superintendências Regionais de Saúde (SRS) e as Gerências Regionais de Saúde (GRS), e que estejam em consonância com a POEPS e com as orientações determinadas no âmbito da Atenção Primária à Saúde, a DPS desenvolveu formulários para o registro dessas experiências e ações (MINAS GERAIS, 2021f).

Inicialmente, os formulários foram desenvolvidos na plataforma de criação de formulário na WEB, destinado ao uso do SUS e de órgãos públicos parceiros, para atividades de interesse público, disponibilizado pelo Departamento de Informática do SUS (DATASUS), intitulado FormSUS. Contudo, devido à independência dos gestores na criação dos formulários e ao risco de exposição de dados inseridos pelos usuários, a plataforma foi descontinuada (BRASIL, 2021).

Portanto, tornou-se necessário a criação dos formulários em outra plataforma que garantisse a continuidade da Comunidade de Conhecimentos e Práticas em Promoção à Saúde de Minas Gerais que tem como objetivo dar visibilidade às ações realizadas pelos municípios, auxiliando os gestores estaduais e municipais e profissionais de saúde em como realizar ações de promoção à saúde (MINAS GERAIS, 2021e).

No intuito de melhorar a visibilidade das ações de educação permanente, desenvolvidas pelas Unidades Regionais de Saúde, a Diretoria de Promoção à Saúde (DPS) construiu o formulário intitulado Educação Permanente em Saúde para o preenchimento com ações de EPS de promoção da saúde direcionadas aos municípios. Além de manter atualizado o registro das qualificações realizadas aos municípios de Minas Gerais pelas regionais de saúde, conforme pactuado na POEPS, não substituindo o registro das atividades, sempre que possível, nos sistemas oficiais da Atenção Primária à Saúde (MINAS GERAIS, 2021f).

Na seleção da plataforma para a criação dos formulários, selecionou-se o Google Forms pelas suas funcionalidades, como perguntas e respostas personalizadas, organização e análises automáticas, possibilidade de adicionar colaboradores e por possibilitar maior acessibilidade. Para apoiar e orientar as regionais em relação ao 
preenchimento dos formulários construiu-se instrutivos para o preenchimento dos formulários eletrônicos da Diretoria.

Figura 01 - Capa dos Instrutivos para o preenchimento dos formulários eletrônicos.

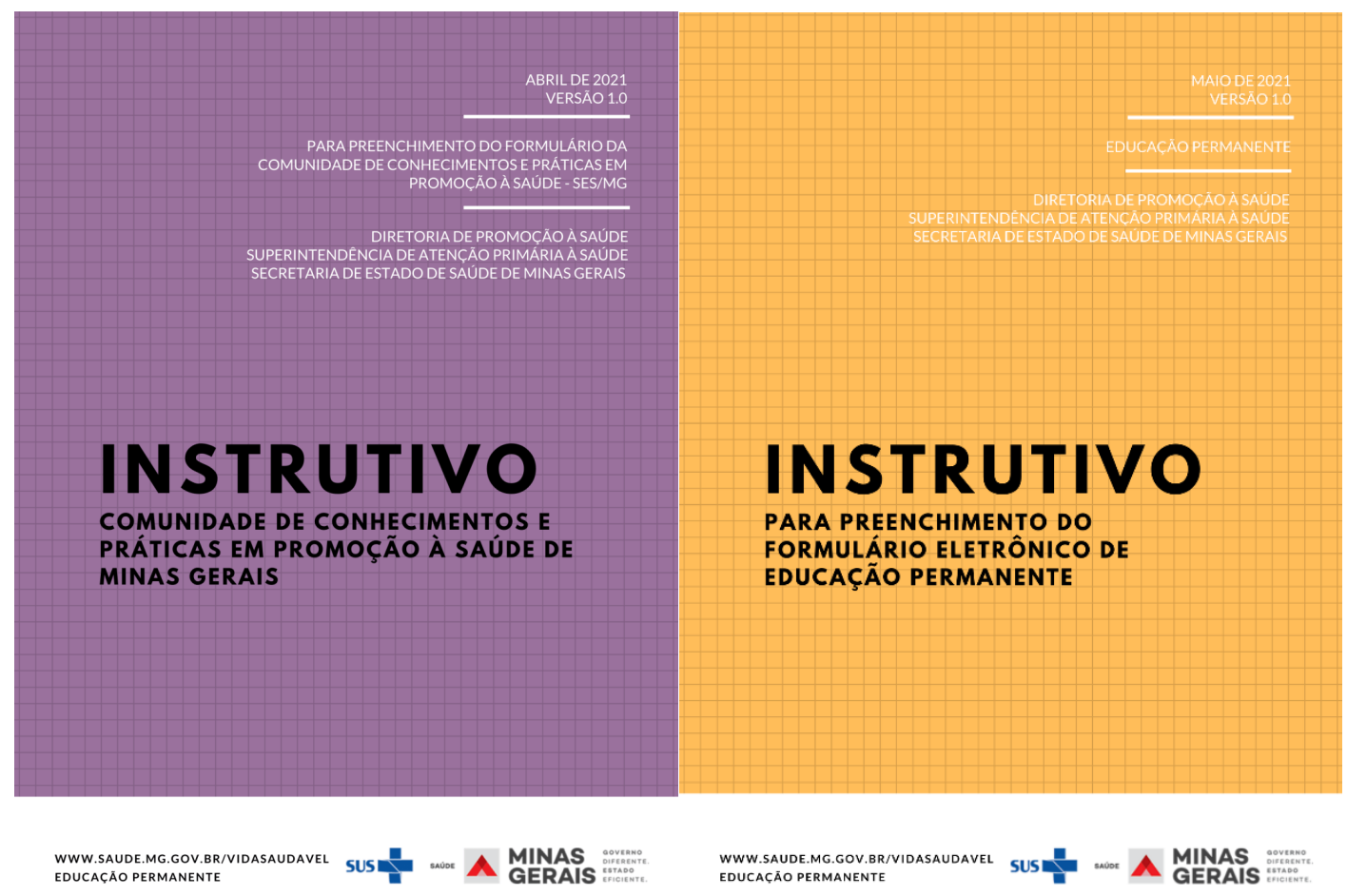

Fonte: Elaboração própria.

Visando reconhecer as ações desenvolvidas pelos municípios que abordassem a promoção da saúde, em consonância com a POEPS, diante do cenário de pandemia da Covid-19, podendo servir de inspiração para novas iniciativas em outros lugares, contribuindo, com isso, para apoiar o desenvolvimento das ações de promoção da saúde pelos municípios, desenvolveu-se o e-book, intitulado: Ações de promoção da saúde em tempos de pandemia pelo COVID-19 (MINAS GERAIS, 2021e). 
Figura 02 - E-book Ações de Promoção da Saúde em tempos de pandemia
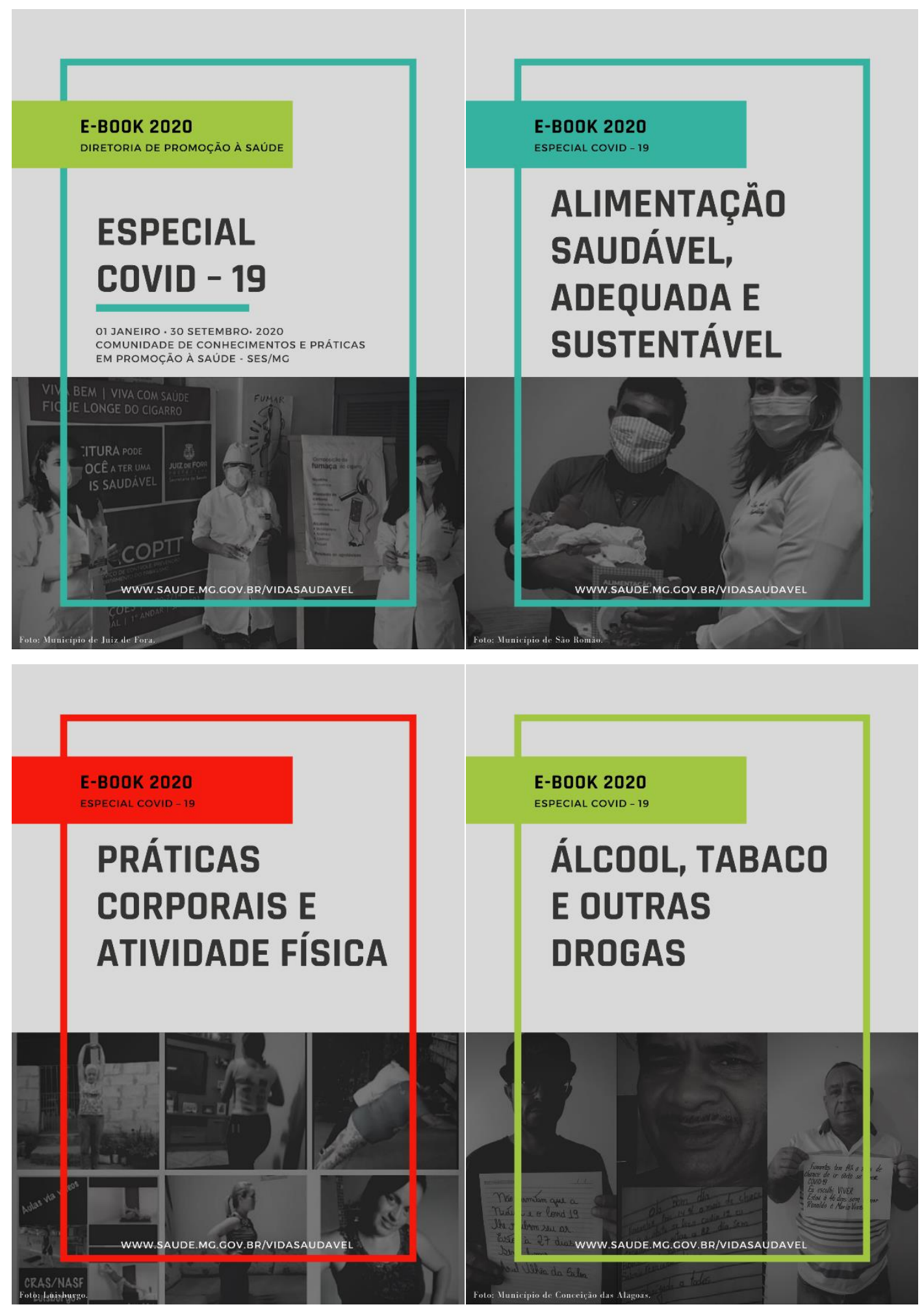

Fonte: Elaboração própria.

Essa experiência reforça que a promoção da saúde é uma estratégia de articulação transversal, em que um conjunto de intervenções individuais, coletivas e ambientais são responsáveis pelas ações intersetoriais sobre os determinantes sociais da saúde, com o 
objetivo de estabelecer mecanismos para reduzir a vulnerabilidade e a desigualdade e aumentar empoderamento social, isto é, encorajar usuários e comunidades a produzir ativamente sua saúde (MINAS GERAIS, 2021f).

\section{CONSIDERAÇÕES FINAIS}

A EPS é um instrumento fundamental para impulsionar a construção e subsidiar espaços de aprendizagem, em que os atores são protagonistas trazendo suas experiências, problemas encontrados nos processos de trabalho na área da saúde, apresentando as verdadeiras necessidades de saúde do território (população) e possibilitando o dialogo coletivo com profissionais de diversas áreas do conhecimento, colaborando para a construção de saberes coletivos.

Além disso, viabiliza a produção de novos pactos e novos acordos coletivos de trabalho no SUS, tendo como foco os processos de trabalho operando no nível coletivo para a viabilização das problematizações e reconhecimento das necessidades de mudança considerando as demandas que emergem de cada localidade.

As diversidades e maneiras inovadoras que os municípios executam as ações da Política de Promoção à Saúde está em constante evolução, e o conhecimento e divulgação dessas ações valorizam o esforço dos municípios e, ainda, auxiliam de forma eficiente outros municípios a desenvolverem ações de promoção da saúde com criatividade e efetividade, em consonância com a POEPS.

A possibilidade da realização do Estágio Curricular Não-Obrigatório na Diretoria de Promoção à Saúde possibilitou a construção de competências e habilidade, como: o desenvolvimento e manuseio de indicadores e sistemas de informação em saúde; responsabilidade sanitária; comportamento organizacional; gestão de pessoas e comunicação nas organizações de saúde; e gestão de conflitos.

\section{AGRADECIMENTOS}

As Referências Técnicas Regionais de Promoção da Saúde das 28 Unidades Regionais de Saúde (URS/SRS/GRS), aos colaboradores Mário Augusto do Rosário Andrade e Beatriz Maria Rodrigues e aos demais servidores da Diretoria de Promoção à Saúde (DPS/SES-MG) pelo apoio e parceria.

\section{REFERÊNCIAS}


BRASIL. Ministério da Saúde. DATASUS retira do ar a ferramenta FormSUS. Departamento de Informática do Sistema Único de Saúde (DATASUS). 2021.

Disponível em: https://datasus.saude.gov.br/datasus-retira-do-ar-a-ferramenta-formsus. Acesso em: 16 jul. 2021.

CORREIA, Maria da Conceição Batista. A observação participante enquanto técnica de investigação. Pensar Enfermagem, v. 13, n. 2, p. 30-36, 2009. Disponível em: http://pensarenfermagem.esel.pt/files/2009_13_2_30-36.pdf. Acesso em: 16 jul. 2021.

MINAS GERAIS. Secretaria de Estado de Saúde. POEPS: Política Estadual de Promoção da Saúde. Subsecretaria de Políticas e Ações de Saúde. Superintendência de Atenção Primária à Saúde. Diretoria de Promoção à Saúde. Belo Horizonte: SES-MG, 2017. 32 p.

MINAS GERAIS. Secretária de Estado de Saúde de Minas Gerais. Missão e Visão. 2020. Disponível em: https://www.saude.mg.gov.br/sobre/institucional/missao-e-visao. Acesso em: 16 jul. 2021.

MINAS GERAIS. Secretária de Estado de Saúde de Minas Gerais. SUS: a SES no SUS. 2021. Disponível em: https://www.saude.mg.gov.br/sus. Acesso em: 16 jul. 2021. a.

MINAS GERAIS. Secretária de Estado de Saúde de Minas Gerais. Programa de Estágio SES. 2021. Disponível em:

https://www.saude.mg.gov.br/component/gmg/page/1765-programa-de-estagio-da-sesmg. Acesso em: 16 jul. 2021. b.

MINAS GERAIS. Secretária de Estado de Saúde de Minas Gerais. Perguntas e Respostas. 2021. Disponível em:

https://coronavirus.saude.mg.gov.br/perguntaserespostas. Acesso em: 16 jul. 2021. c.

MINAS GERAIS. Secretaria de Estado de Saúde. Guia Orientador da Atenção Primária à Saúde (APS) de Minas Gerais para o Enfrentamento da Pandemia de COVID-19. 5. ed. Belo Horizonte: SES-MG, 2021. 89 p. d.

MINAS GERAIS. Secretaria de Estado de Saúde. E-Book Especial Covid-19.

Subsecretaria de Políticas e Ações de Saúde. Superintendência de Atenção Primária à Saúde. Diretoria de Promoção à Saúde. Belo Horizonte: SES-MG, 2021. 109 p. e.

MINAS GERAIS. Secretária de Estado de Saúde de Minas Gerais. Vida Saudável. 2021. Disponível em: https://www.saude.mg.gov.br/vidasaudavel. Acesso em: 16 jul. 2021. f.

SPRADLEY, James P. Participant Observation. Administrative Science Quarterly, v. 25, n. 3, p. 526, set. 1980. JSTOR. http://dx.doi.org/10.2307/2392270.

XU, Zhe et al. Pathological findings of COVID-19 associated with acute respiratory distress syndrome. The Lancet Respiratory Medicine, v. 8, n. 4, p. 420-422, abr. 2020.

Recebido em: 15/01/2022

Aprovado em: 16/02/2022

Publicado em: 18/02/2022 